\title{
DEVIATIONS FROM THE CRITICAL STATE MODEL OBSERVED IN NIOBIUM
}

\author{
E.A. GIJSBERTSE and L.J.M. van de KLUNDERT \\ Twente University of Technology, Department of Applied Physics, \\ 7500 Ae Enschede, The Netherlands
}

Received 10 March 1980

\begin{abstract}
The values of the critical current density of niobium slabs, measured with the help of trapezoidal ac magnetic fields are not in agreement with the critical state model. Extensions of this model are suggested to explain the observed deviation.
\end{abstract}

Introduction. In a generalized critical state model (CSM) $[1,2]$ the pinning of flux lines in type II superconductors is described by a critical current density $j_{\mathrm{c}}$, which may depend on magnetic induction $B$ and on position. Additional screening of flux by surface currents is expressed in terms of a $B_{\text {en }}\left(B_{\mathrm{a}}\right)$ relation in the case of an increasing external induction and of $B_{\text {ex }}\left(B_{\mathrm{a}}\right)$ in the decreasing case. $B_{\text {en }}\left(B_{\mathrm{a}}\right)$ and $B_{\text {ex }}\left(B_{\mathrm{a}}\right)$ give the value of $B$ just inside a narrow surface layer as a function of external field. Finally, under ac conditions also flux flow effects have to be considered; they will be described in the same way as normal currents in terms of a flux flow conductivity $\sigma_{\mathrm{ff}}$.

An inductive technique making use of a trapezoidal ac field (amplitude $b_{0}$ ) superposed on a static field $B_{0}$ has proven to be a powerful instrument for the study of the various parameters of the CSM [1]. A careful analysis of the waveform of the induced voltage leads to a separation of the various contributions to the ac losses ${ }^{\neq 1}$. In fact, it is possible to determine the flux distribution inside a superconductor, provided that flux flow effects may be neglected and that $j_{\mathrm{c}}$ is independent of $B$ within the applied ac field range. In this case the voltage induced in a pick-up coil around a superconducting slab (thickness $2 d$ ) in a parallel increasing field is given by:

$E=\left(d-x_{\mathrm{t}}\right)\left(\mathrm{d} B_{\text {en }} / \mathrm{d} B_{\mathrm{a}}\right)\left(\mathrm{d} B_{\mathrm{a}} / \mathrm{d} t\right)$,

where $x_{\mathrm{t}}$ is the position to which the flux change has entered. The position of this flux front can be determined from:

$$
E / E_{1}=\left(d-x_{\mathrm{t}}\right) / d,
$$

where $E_{1}$ is the induced voltage at the same external field, when the flux front has reached the centre plane of the slab $\left(x_{\mathrm{t}}=0\right) ; E_{1}$ is independent of the external field at which the field change started and depends only on $B_{\mathrm{a}}$. The local magnetic induction at this position is given by:

$$
\begin{aligned}
& B\left(x_{\mathrm{t}}, B_{\mathrm{a}}\right)=\frac{1}{2}\left[B_{\mathrm{ex}}\left(B_{0}-b_{0}\right)+B_{\mathrm{en}}\left(B_{\mathrm{a}}\right)\right] \\
& \quad=B_{\mathrm{ex}}\left(B_{0}-b_{0}\right)+\frac{1}{2}\left(B_{\mathrm{a}}-B_{\mathrm{a}_{0}}\right) \mathrm{d} B_{\mathrm{en}} /\left.\mathrm{d} B_{\mathrm{a}}\right|_{B_{\mathrm{a}_{0}}} ;
\end{aligned}
$$

$B_{\mathrm{a}_{0}}$ is defined by $B_{\mathrm{en}}\left(B_{\mathrm{a}_{0}}\right)=B_{\mathrm{ex}}\left(B_{0}-b_{0}\right)$. With the help of these equations the flux distribution at the moment at which the external field change started can be determined. An analogous derivation can be given for a decreasing field.

Experimental results. The experimental method described above has been used to study the critical state in a $\mathrm{Nb}$ slab. The sample $\left(20 \times 5 \times 0.3 \mathrm{~mm}^{3}\right)$ was taken from commercial $\mathrm{Nb}$ plate and subsequently annealed. Experiments were done with trapezoidal ac fields of various amplitudes and $\mathrm{d} B_{\mathrm{a}} / \mathrm{d} t$ values superposed on a static field. A more detailed description of the experimental equipment is given elsewhere ${ }^{\ddagger 1}$. A number of normalized $E$ versus $B_{\mathrm{a}}$ curves for in-

\$1 A more detailed discussion of the experimental method and the equipment which is used for these measurements will be published in a subsequent paper. 

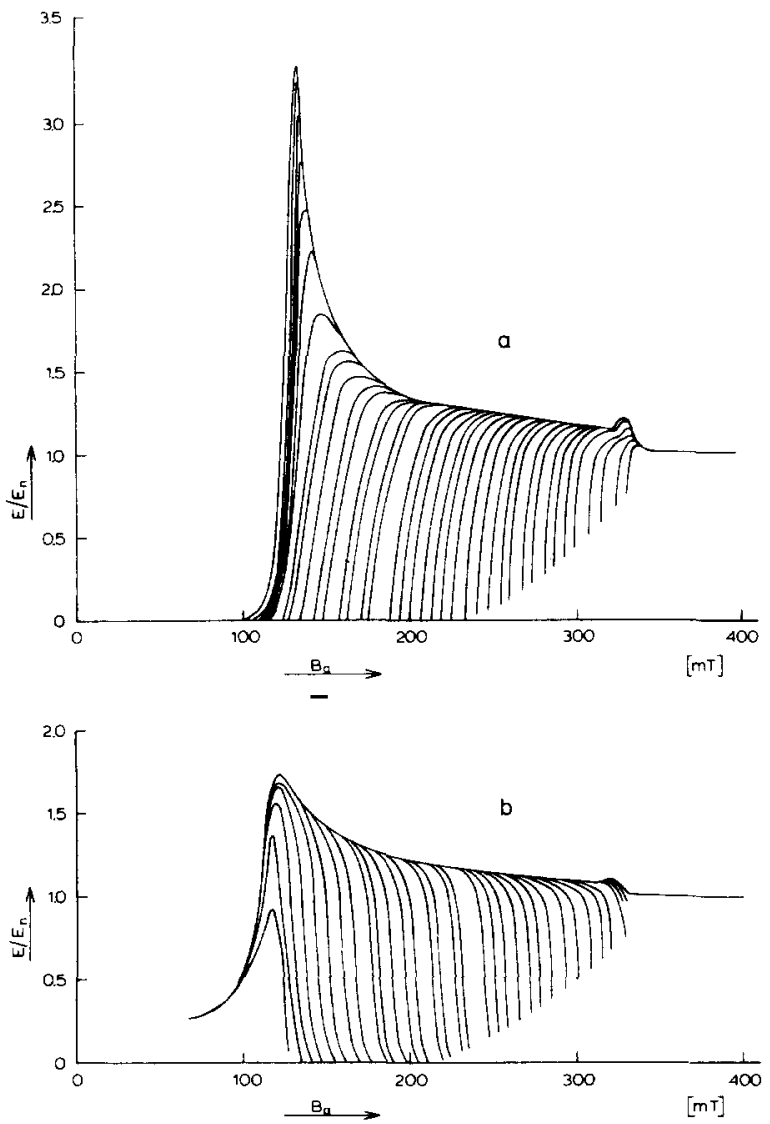

Fig. 1. A set of normalized $E$ versus $B_{\text {a }}$ curves in increasing (a) and in decreasing (b) external field for a Nb slab $\left(b_{0}=\right.$ $32 \mathrm{mT}, \mathrm{d} B_{\mathrm{a}} / \mathrm{d} t=0.67 \mathrm{~T} / \mathrm{s}$ ).

creasing and decreasing fields are given in fig. 1 . The point at which an individual curve reaches the envelope indicates the penetration of the flux front to the centre of the slab; the envelope curves give the voltages $E_{1}\left(B_{\mathrm{a}}\right)$ which are a measure for $\mathrm{d} B_{\mathrm{en}} / \mathrm{d} B_{\mathrm{a}}$ and $\mathrm{d} B_{\text {ex }} / \mathrm{d} B_{\mathrm{a}}$, respectively. A very remarkable feature is that in decreasing field the centre plane is reached much earlier than in increasing field. This suggests a smaller value of $j_{\mathrm{c}}$ in decreasing field. This is confirmed by the results in fig. 2 where the value of $j_{\mathrm{c}}$ is plotted as a function of $B$. The $j_{\mathrm{c}}$ values are determined from the linear middle part of the $E$ versus $B_{\mathrm{a}}$ curves, according to eqs. (2) and (3). Here also the difference between $j_{\mathrm{c}}$ in increasing and decreasing fields is observed.

Most experiments were done at a temperature of $4.2 \mathrm{~K}$ but experiments at $2.0 \mathrm{~K}$ exhibit the same fea-

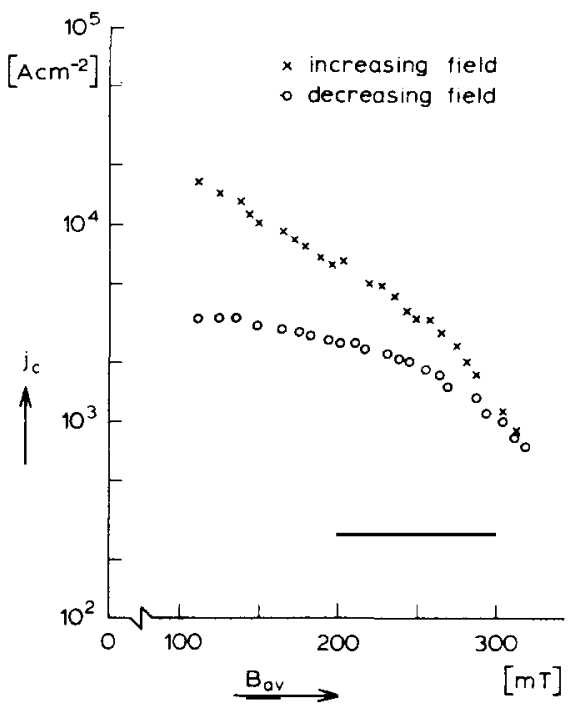

Fig. 2. The critical current density $j_{\mathrm{c}}$, measured in increasing and decreasing field, plotted versus the average internal induction $B_{\text {av. }}$

tures. Experiments performed in the same way on a $\mathrm{Nb}-50 \%$ Ti sample did not show this phenomenon [3].

Discussion. The apparent difference in $j_{\mathrm{c}}$ values cannot be explained by assuming that $j_{\mathrm{c}}$ is actually different in both directions because the method fundamentally cannot distinguish between the two values. This is a consequence of the fact that the induced voltage is determined equally by both the flux profile in increasing and decreasing field. Additional confirmation of this point is given by the experimental result that the $j_{\mathrm{c}}$ values are independent of field reversal. Also the fact that $j_{\mathfrak{c}}$ depends on $B$ cannot give any explanation of the observed phenomenon; it can be seen easily that in increasing field a smaller value of $j_{\mathrm{c}}$ should be obtained because the average value of $B$ inside the sample is larger in this case than in decreasing field. With respect to the behaviour of surface screening currents no special assumptions were made. A small correction to the results can be obtained by taking into account the second order term in the Taylor expansion of eq. (3); this correction, however, is not enough to explain the large difference in $j_{\mathrm{c}}$ values. Although flux flow effects may not be neglected in this sample, they are not responsible for the observed phenomenon: the $j_{\mathrm{c}}$ values are independent of the 
applied $\mathrm{d} B_{\mathrm{a}} / \mathrm{d} t$ in a wide range of $\mathrm{d} B_{\mathrm{a}} / \mathrm{d} t$ values $(0.67$ $\mathrm{T} / \mathrm{s}$ to $27 \mathrm{~T} / \mathrm{s}$ ). A decrease of the flux flow conductivity $\sigma_{\text {ff }}$ with increasing $B$ (which is commonly observed) will lead to an apparently larger value of $j_{\mathrm{c}}$ in decreasing field, which is in contradiction with the experimental results.

Conclusively it can be said, that the observed difference in $j_{\mathrm{c}}$ values cannot be explained in terms of the generalized critical state model, since none of the parameters of this model nor their $B$-dependences can account for this behaviour. So far a realistic explanation cannot be given. Several deviations from the CSM may be considered. First of all the model may be extended to a non-isothermal CSM. In this case the temperature of the sample varies during one period of the ac field due to dissipation; now also the temperature dependence of the various parameters must be taken into account. A second extension concerns the inclusion of forces on flux lines due to temperature gradients inside the sample [4]. These thermomagnetic forces are directed towards the lower temperature region. Numerical solution of the heat diffusion equation for a slab with a dissipation distribution similar to the one in a superconducting slab with constant pinning force in an ac magnetic field indicates a temperature profile with a high temperature at the position of the flux front and a lower temperature at the surface. The result of this temperature gradient is a force on the flux lines towards the surface. In increasing field this means that flux penetrates the slab less easily, i.e., a larger critical current density while in decreasing field flux moves out more easily, i.e., a lower $j_{\mathrm{c}}$ value. So thermo-magnetic effects might give an explanation of the experimental data. Finally when these two possibilities cannot be held responsible for the observed results, a more fundamental breakdown of the CSM in which also values of the supercurrent between $\pm_{c}$ may exist for a finite time interval, may be considered. A more extensive presentation of the results obtained on this sample will be given in a subsequent paper.

\section{References}

[1] L.J.M. van de Klundert, E.A. Gijsbertse and H.P. van de Braak, Physica 94B (1978) 41.

[2] J.R. Clem, J. Appl, Phys. 50 (1979) 3518.

[3] E.A. Gijsbertse, Proc. Intern. School on Superconductivity (Piechowice, Poland), to be published.

[4] F.A. Otter and P.R. Solomon, Phys. Rev, Lett. 16 (1966) 681 . 